\title{
Estudio sobre las relaciones del síndrome de burnout con algunos factores psicosociales
}

Andrea López Fernández, Damián Sáez Rodríguez, María Jesús García Aranda, Alba López Paiz y Laura Hernández Saavedra. Universidad de Granada

Recepción: 12 de mayo de 2015 | Revisión: 17 de junio de 2015 | Aceptación/Publicación: 17 de julio de 2015

Citar: Lopez, A., Saez, D., Garcia, MJ., Lopez, A. y Hernandez, L. (2015). Estudio sobre las relaciones del síndrome de burnout con algunos factores psicosociales. ReiDoCrea, 4, 94-99.

Resumen: El Síndrome de Burnout es un problema psicosocial relevante porque el trabajador pierde su capacidad de motivación por el trabajo, su rendimiento laboral es bajo y se deteriora su salud física y mental, por eso se considera que es un problema importante sobre todo en el ámbito penitenciario, donde los trabajadores están sometidos a constantes presiones. El objetivo de este estudio es analizar la relación existente entre Burnout, autoestima, género y experiencia laboral en funcionarios de prisiones. El estudio se realizó con 34 funcionarios penitenciarios de la cárcel de Albolote (Granada). Los instrumentos utilizados fueron: la Escala de Autoestima de Rosenberg (1965) y el Cuestionario de Maslach Burnout Inventory (1986). Los resultados obtenidos muestran que no existen relaciones significativas entre Burnout, autoestima, experiencia laboral y género; aunque se han encontrado algunas diferencias de género en las variables de estudio.

Palabras clave: Burnout, género, experiencia laboral, autoestima, despersonalización, cansancio emocional y realización personal.

\section{Introducción}

En los últimos años se ha puesto de relieve cómo muchos profesionales se ven sometidos continuamente a unas condiciones laborales de alta implicación emocional, a fuertes demandas interpersonales que exigen reaccionar y adaptarse rápidamente en las experiencias de estrés que, de no afrontarse adecuadamente (Hernández Zamora, Olmedo Castejón e Ibáñez Fernández, 2004), pueden llevar a estas personas a padecer el Síndrome de Burnout (Maslach, 2003; Maslach, Schaufeli y Leiter, 2001) o "síndrome de quemarse por el trabajo", la expresión más apropiada a utilizar en castellano según Gil-Monte (2005).

El concepto Burnout como fenómeno psicológico tiene su origen en Estados Unidos, asociándose a trabajos realizados a mediados de la década de los setenta para dar una explicación al proceso de deterioro en los cuidados y atención profesional a los usuarios de las organizaciones de servicios. Actualmente parece haber acuerdo en la aceptación de que el Burnout es un síndrome tridimensional de agotamiento emocional, despersonalización y pérdida de realización personal en el trabajo. La dimensión de agotamiento emocional, considerada por Maslach (1993; 1999) como la más próxima a una variable de estrés, se caracteriza por sentimientos de desgaste y agotamiento de los recursos emocionales. Es un sentimiento de que nada se puede ofrecer a los demás. La despersonalización se refiere al sentimiento de endurecimiento emocional, desapego, desarraigo, pérdida de la capacidad de contacto y a la adopción de actitudes negativas, frías y distanciadas hacia los receptores de los servicios. Por último, la falta de realización personal corresponde a la aparición de sentimientos negativos de inadecuación, falta de competencia y eficacia profesional, disminución de las expectativas personales, que implica una autoevaluación negativa donde se puede desarrollar rechazo a sí mismo y hacia los logros personales, así como sentimientos de fracaso y baja autoestima. Aunque el Burnout se ha estudiado tradicionalmente en profesiones de ayuda, actualmente se ha verificado una ampliación del concepto de Burnout a todas las profesiones en general. Un marco 
decisivo en ese sentido fue la publicación del instrumento Maslach Burnout Inventory General Survey (MBI-GS) (Schaufeli, Leiter, Maslach y Jackson, 1996) susceptible de aplicar a otras profesiones más allá de las de ayuda y que ha mostrado que la estructura tridimensional del Burnout se mantiene estable en profesiones.

El Síndrome de Burnout es un problema psicosocial relevante porque el trabajador pierde su capacidad de motivación por el trabajo, su rendimiento laboral es bajo y se deteriora su salud física (e.g., Moya-Albiol, Serrano, González-Bono, RodríguezAlarcón y Salvador, 2005) y mental (Sandstrom, Rhodin, Lundberg, Olsson y Nyberg, 2005).

Uno de los contextos laborales que tiene suficientes componentes objetivos altamente estresantes es el penitenciario (Anson y Bloom, 1988; Clemente, 1995; Garrido, 1985; Martínez, 1995). Además de esta particularidad, se dan una serie de características propias que le hacen adquirir una dimensión difícilmente comparable a otros desempeños laborales. Algunas de estas características específicas son: masificación en los centros; escasa disposición de los internos a colaborar con el funcionario (en muchos casos franca oposición); hostilidad y existencia de riesgo a la integridad física; escasa valoración social del trabajo realizado; y el contagio de la "cultura de la prisión" (Caballero 1986). Existen estudios que han comprobado la existencia de Burnout en los funcionarios de prisiones (e.g., Hurst y Hurst, 1997; Morgan, Van Haveren y Pearson, 2002; Schaufeliy Peeters, 2000). Otros estudios en funcionarios de prisiones reflejados por Moon y Maxwell (2004) manifiestan que altos niveles de estrés llevan a una insatisfacción laboral, enfermedades psicológicas, Burnout o problemas familiares. Existen evidencias que relacionan el estrés, la tensión, el propio síndrome de agotamiento profesional y el mobbing (acoso psicológico en el trabajo) con una baja autoestima. La variable autoestima, es conocida como el recurso natural que proporciona al sujeto la condición de tener confianza en sí mismo. La autoestima nos proporciona "confiar en nuestros procesos de razonamiento, comprensión, elección, decisión y de acción" (Bernal 1995).

El objetivo principal de este estudio es analizar la relación entre el Síndrome de Burnout y algunos factores psicosociales como autoestima, género y experiencia laboral. En concreto se propone someter a prueba en primer lugar, que altos niveles en el Síndrome de Burnout reflejan menores puntuaciones en autoestima; en segundo lugar se cree que el género masculino presenta un mayor nivel de síndrome de Burnout en comparación con el género femenino (dado que se asocia el género femenino con una mayor capacidad de resistencia a la presión, hablando en términos generales); y por último, a más experiencia laboral, menores niveles el Síndrome de Burnout debido a que se adquiere mayor control y por lo tanto la sensación de "estar quemado en el trabajo" disminuye.

\section{Método}

\section{Participantes}

Se seleccionaron como participantes a 34 funcionarios de prisión de la prisión de Albolote (Granada), de los cuales 18 son hombres y 16 mujeres. Los participantes tenían una edad comprendida entre 35 y 62 años y una experiencia laboral entre 11 y 37 años. 


\section{Instrumentos}

La autoestima fue evaluada mediante La Escala de Autoestima de Rosenberg (1965). Esta prueba ha sido traducida a 28 idiomas, y validada interculturalmente en 53 países. Está formada por 10 ítems en los cuales las personas evaluadas deben de elegir una de las 4 opciones de respuesta posibles (Muy de acuerdo, De acuerdo, En desacuerdo y Muy en desacuerdo). Para la corrección de la prueba siguiendo las instrucciones del autor, se le asignaron valores numéricos a las posibles respuestas [Muy de acuerdo (1), De acuerdo (2), En desacuerdo (3) y Muy en desacuerdo (4)].

El Síndrome de Burnout fue evaluado mediante el Cuestionario de Maslach Burnout Inventory (1986). Este test pretende medir la frecuencia y la intensidad con la que se sufre el Burnout. Está constituido por 22 ítems en forma de afirmaciones, sobre los sentimientos y actitudes del profesional en su trabajo. Mide los tres aspectos del síndrome:

- Subescala de agotamiento emocional. Consta de 9 preguntas. Valora la vivencia de estar exhausto emocionalmente por las demandas del trabajo. Puntuación máxima 54. Esta subescala obtuvo una fiabilidad de .848 por el Alfa de Cronbach.

- Subescala de despersonalización. Está formada por 5 ítems. Valora el grado en que cada uno reconoce actitudes de frialdad y distanciamiento. Puntuación máxima 30 . Esta subescala obtuvo una fiabilidad de .537 por el Alfa de Cronbach.

- Subescala de realización personal. Se compone de 8 ítems. Evalúa los sentimientos de autoeficacia y realización personal en el trabajo. Puntuación máxima 48. Esta subescala obtuvo una fiabilidad de .723 por el Alfa de Cronbach.

\section{Procedimiento}

Se trata de un estudio correlacional en el que los datos fueron recogidos de manera aleatoria durante varios días, en el Centro Penitenciario de Albolote, ubicado en la ciudad de Granada (España).

Los participantes debían contestar ambos cuestionarios (como hemos dicho previamente, integrados) de manera anónima y tenían que indicar otras variables como son la edad, el estado civil, el género y los años de experiencia laboral. Una vez recogidos los cuestionarios, se procedió al análisis de los datos mediante el programa IBM SPSS Statistics.

Para comparar las medias de los resultados obtenidos entre hombres y mujeres, se aplicó la prueba T-Student para muestras independientes. A continuación, se dividieron los datos en hombres y mujeres y se realizaron pruebas correlacionales entre las variables autoestima y experiencia laboral, y las tres variables del síndrome de burnout (agotamiento emocional, despersonalización y realización personal).

\section{Resultados}

Para comprobar si hay diferencias de género en hombres y mujeres en las variables psicosociales objeto de estudio, podemos observar en la Gráfica 1, que no existen diferencias significativas de género; sin embargo tal y como se puede observar, en comparación con las mujeres, los hombres obtienen puntuaciones mayores en tres de las cuatro variables: autoestima, despersonalización y realización personal; por el contrario obtienen menor puntuación en cansancio emocional. 


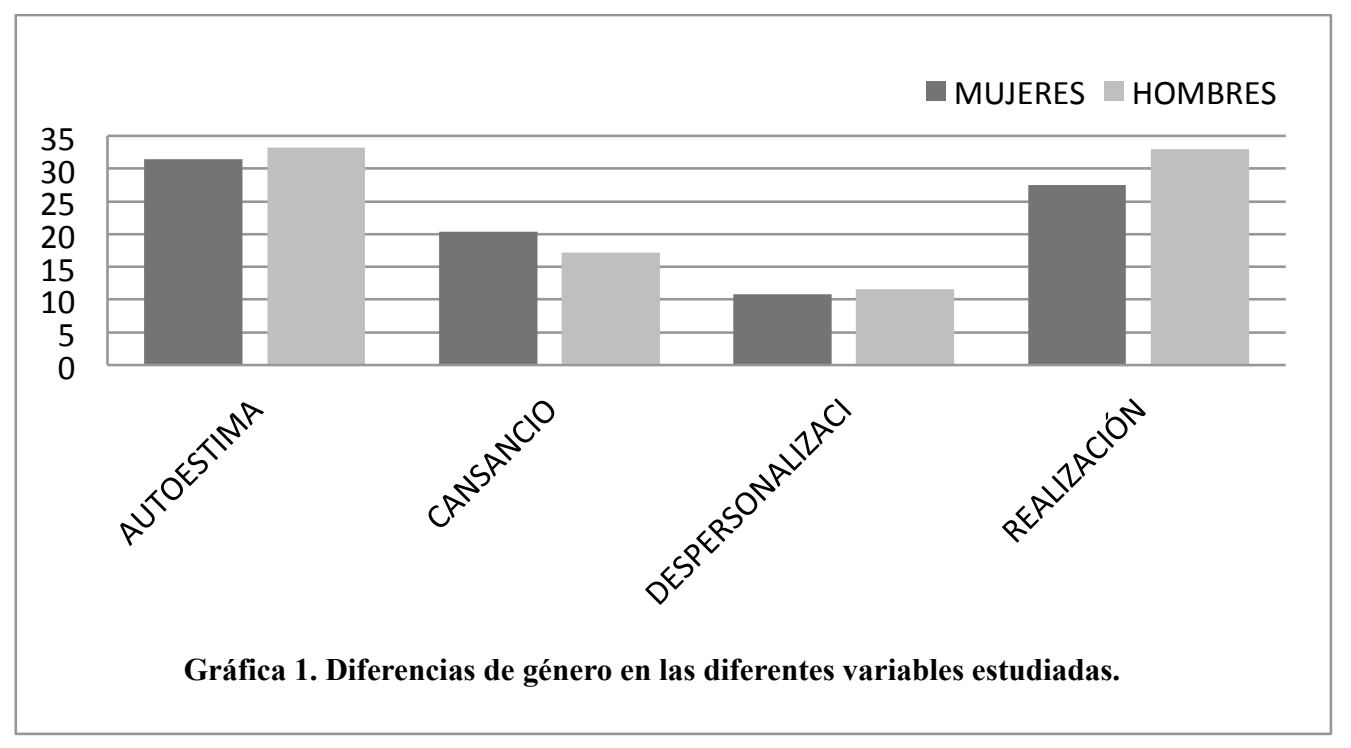

Tras analizar las correlaciones bivariadas realizadas en la muestra segmentada (hombres y mujeres), y cuyos resultados se muestran en la Tabla 1, se puede observar que no hay una correlación significativa entre las puntuaciones en Síndrome de Burnout y autoestima; de igual forma que no existe dicha relación entre Burnout y experiencia laboral. No obstante, sí que se encontraron correlaciones significativas en algunas variables en hombres pero no en mujeres. Concretamente se observa una correlación positiva entre despersonalización y cansancio emocional, una correlación negativa entre cansancio emocional y realización personal, y una correlación negativa entre despersonalización y realización personal.

\begin{tabular}{|c|c|c|c|c|c|c|c|c|c|c|}
\hline \multicolumn{11}{|c|}{$\begin{array}{c}\text { Tabla } 1 \\
\text { Correlaciones bivariadas entre las diferentes variables }\end{array}$} \\
\hline & \multicolumn{2}{|c|}{1} & \multicolumn{2}{|c|}{2} & \multicolumn{2}{|c|}{3} & \multicolumn{2}{|c|}{4} & \multicolumn{2}{|c|}{5} \\
\hline & $M$ & $H$ & $M$ & $H$ & $M$ & $H$ & $M$ & $H$ & M & $H$ \\
\hline 1. EXP LAB & --- & --- & -.109 & -.403 & -.392 & .063 & -.114 & -.095 & 261 & -.112 \\
\hline 2. AUTOEST & -.109 & -.403 & --- & --- & .332 & -.195 & .082 & -.012 & -.059 & -.158 \\
\hline 3.CE (B) & -.392 & .063 & .332 & -.195 & --- & --- & .381 & $.673^{\star *}$ & -.442 & $-.447^{*}$ \\
\hline 4.DES (B) & -.114 & -.095 & .082 & -.012 & .381 & $.673^{* \star}$ & --- & --- & .114 & $-.498^{*}$ \\
\hline 5. RE PE (B) & .261 & -.112 & -.059 & -.158 & -.442 & $-.447^{*}$ & .114 & $-.498^{*}$ & --- & --- \\
\hline $\begin{array}{l}\text { Nota: EXP LAB: } \\
\text { personal } \\
{ }^{*} p<0.05^{* *} p<0 .\end{array}$ & & IUTOES & 4 & CE (B): & & ional; DE & B): des & onalizació & RE PE (E & realización \\
\hline
\end{tabular}

\section{Discusión}

El Síndrome de Burnout es un problema en la actualidad debido a las repercusiones que tiene en los trabajadores que lo padecen (Hernández Zamora, Olmedo Castejón e Ibáñez Fernández, 2004). Por ello, el objetivo de esta investigación consistió en analizar las relaciones del Síndrome con algunas variables psicosociales como son experiencia laboral, género y autoestima.

A la vista de los resultados obtenidos no se puede concluir que funcionarios de prisiones con puntuaciones altas en Burnout se asocie con tener niveles bajos de autoestima -en contraposición con resultados obtenidos en estudios anteriores (Moon y Maxwell, 2004)-; ni que el sexo masculino sufra más Burnout que el femenino 
(aunque hay ligeras diferencias en las puntuaciones obtenidas en las dimensiones del síndrome). Por último, tampoco podemos afirmar que cuantos más años de experiencia laboral tengan los trabajadores, sufrirán menos Burnout, por lo que no se puede concluir, como se esperaba, que éste sea un factor de protección (esto es, a mayor cantidad de años trabajados, mayor control y menor vulnerabilidad ante el síndrome).

Una vez resueltas las hipótesis principales de la investigación, se estudiaron las diferencias entre hombres y mujeres en la relación entre las variables: autoestima, experiencia laboral y las tres variables que componen el Burnout (cansancio emocional, despersonalización y realización personal). Al analizar estos resultados se observaron correlaciones significativas, tales como que los hombres que tenían puntuaciones altas en cansancio emocional, también tenían puntuaciones altas en despersonalización; así como que puntuaciones altas en cansancio emocional, se correspondían con puntuaciones bajas en realización personal; por último, puntuaciones altas en despersonalización, también se asocian a puntuaciones bajas en realización personal. Todos estos resultados sólo se observaron en hombres, mientras que en mujeres no se observó ninguna relación significativa entre las variables objeto de estudio (Tabla1).

Los resultados obtenidos podrían explicarse por las posibles limitaciones del presente estudio en lo que a la metodología se refiere. Dichas limitaciones son algunos aspectos que no se han controlado y que han podido influir en los resultados tales como la heterogeneidad de la muestra, ya que la experiencia laboral no ha sido distribuida de manera uniforme; y el tamaño limitado de ésta, debido al reducido número de participantes. Por todo ello, es difícil sacar conclusiones definitivas y generales de una muestra tan pequeña.

Para futuros estudios sería conveniente controlar las variables de una forma más rigurosa y obtener un mayor número de participantes, ya que se estima que el síndrome de Burnout y las variables que influyen en él son un factor muy importante a estudiar, con el propósito de mejorar las calidades de la relación y la satisfacción en un ámbito tan imprescindible en la vida de toda persona como es el trabajo.

\section{Referencias}

Hernández Zamora, G.L., Olmedo Castejón, E. e Ibáñez Fernández, I. (2004). Esta quemado (burnout) y su relación con el afrontamiento. International Journal of Clinical and Health Psychology, 4, 323-336.

Maslach, C. (2003). Job burnout: New directions in research and intervention. Current Directions in Psychological Science, 12, 189-192.

Gil-Monte, P.R. (2005). El síndrome de quemarse por el trabajo (Burnout): una enfermedad laboral en la sociedad del bienestar. Madrid: Pirámide.

Moya-Albiol, L., Serrano, M.A., González-Bono, E., Rodríguez-Alarcón, G. y Salvador, A. (2005). Respuesta psicofisiológica de estrés en una jornada laboral. Psicothema,17, 205-211.

Maslach, C. y Jackson, S. E. (1981). The Maslach Burnout Inventory. Research Edition. Palo Alto, CA: Consulting Psychologists Press.

Jackson, S. E. (1996). Maslach Burnout Inventory General Survey. In Maslach, C., Sandstrom, A., Rhodin, I.N., Lundberg, M., Olsson, T. y Nyberg, L. (2005). Impaired cognitive performance in patients with chronic burnout syndrome. Biological Psychology, 69, 271- 279.

Anson, R.H. y Bloom, M.E. (1988). Police stress in an occupational context. Journal of Police Science and Administration, 16, 229-235. 
Caballero Romero, J. J. (1986). El mundo de los presos. En F. Jiménez Burillo y M.Clemente (Comps.), Psicología social y sistema penal (págs. 269-301). Madrid: Alianza Editorial.

Schaufeli, W.B. y Peeters, M.C.W. (2000). Job stress and burnout among correctional officers: A literature review. International Journal of Stress Management, 7, 19-49.

Hurst, T.E. y Hurst, M.M. (1997). Gender differences in mediation of severe occupational stress among correctional officers. American Journal of Criminal Justice, 22, 121-137.

Morgan, R.D., Van Haveren, R.A. y Pearson, C.A. (2002). Correctional officer burnout. Further analyses. Criminal Justice and Behavior, 29, 144-160.

Moon, B. y Maxwell, S.R. (2004). The sources and consequences of correctional officer' stress: A South Korean example. Journal of Criminal Justice, 32, 359-370.

Bernal L. (1995). La satisfacción de los directivos con su trabajo: su autoestima. Organización y Gestión Educativa, 2, 3-7. 